\title{
SUMS OF THREE INTEGER SQUARES IN COMPLEX QUADRATIC FIELDS
}

\author{
DENNIS R. ESTES AND J. S. HSIA ${ }^{1}$
}

\begin{abstract}
We classify all complex quadratic number fields that have all their algebraic integers expressible as a sum of three integer squares. These fields are $F=\mathbf{Q}(\sqrt{-D}), D$ a positive square-free integer congruent to $3(\bmod 8)$ and such that $D$ does not admit a positive proper factorization $D \equiv d_{1} d_{2}$ that satisfies simultaneously: $d_{1} \equiv 5,7(\bmod 8)$ and $\left(d_{2} / d_{1}\right)=1$.
\end{abstract}

We consider the following problem: Which complex quadratic number fields $F=$ $\mathbf{Q}(\sqrt{-D}), D$ square-free, have all their algebraic integers expressible as a sum of three integer squares?

The fact that all the algebraic integers in $F$ must be representable as a sum of 3 integer squares forces some necessary conditions on $D$. Firstly, -1 must be so expressible implies that -1 is a sum of two squares in $F$. This is because the minimal number ("Stufe") of representing -1 as a sum of squares in any formally nonreal field is well known to be always a 2-power. On the other hand, those number fields with Stufe $\leq 2$ are known, already to Hasse in [2], to be classified by the property that all the local degrees are even at all the primes lying over $2 \mathbf{Z}$. In the present situation this means $D \neq 7(\bmod 8)$. Secondly, since every sum of squares is congruent to a perfect square $\bmod 2$, we see that if $2 \mathbf{Z}$ were ramified in $F$ then there are algebraic integers which are incongruent to a square mod 2. Therefore, the discriminant of $F$ must be an odd integer. These conditions then force $2 \mathbf{Z}$ to be inert in $F$, i.e., $D \equiv 3(\bmod 8)$, which we shall assume henceforth.

Presently, the following partial results are known. Every integer in $F$ may be expressible as a sum of 3 integer squares when any of the following conditions is fulfilled:

(i) the class number $h_{F}$ of $F$ is odd [3, p. 532];

(ii) $h_{F}=2 \times$ (odd integer) $[\mathbf{1}]$;

(iii) $D=q_{1} q_{2} q_{3}$ where $q_{i}$ are primes congruent to $3(\bmod 4)$ and $\left(q_{i} q_{j} / q_{k}\right)=-1$ for $\{i, j, k\}=\{1,2,3\}[\mathbf{1}]$

(iv) $X^{2}+2 Y^{2}=D$ is solvable in $\mathbf{Z}[\mathbf{6}, \mathrm{p} .7]$.

In terms of factorizations of $D$, the classical theorems of Gauss and Rédei-Reichardt imply respectively that (i) is equivalent to $D$ being a prime and (ii) to $D=p q$ where $p, q$ are primes and $(p / q)=-1$. As for (iv) it is well known that the only odd square-free positive integers (and hence $D$ ) which can be represented by the binary form $X^{2}+2 Y^{2}$ are those whose prime factors are either congruent to 1 or to 3 $(\bmod 8)$.

Received by the editors October 15, 1982.

1980 Mathematics Subject Classification. Primary 10C02, $10 \mathrm{C} 05$.

Key words and phrases. Exceptional integer, genus, $\chi$-invariant, Artin symbol.

${ }^{1}$ Research partially supported by NSF Grant MCS 80-02985 and MCS 82-02065.

(C) 1983 American Mathematical Society $0002-9939 / 83 \$ 1.00+\$ .25$ per page 
In fact, it was shown in [1] that in the first three cases above every algebraic integer in $F$ is representable by every quadratic form in the genus of $X^{2}+Y^{2}+X^{2}$.

On the negative side, however, one knows not every integer is a sum of 3 integer squares for certain fields whose ideal class groups are either the Klein 4-group or the cyclic group of order four. See [1].

We shall give a complete answer to our problem, which is the following

TheOREM. Every algebraic integer in $F=\mathbf{Q}(\sqrt{-D}), D$ a positive square-free integer, can be expressed as a sum of three integer squares when and only when $D \equiv$ $3(\bmod 8)$ and $D$ does not admit a positive proper factorization $D=d_{1} d_{2}\left(\right.$ i.e., $\left.d_{i}>1\right)$ which satisfies the conditions: $(1) d_{1} \equiv 5,7(\bmod 8)$ and $(2)\left(d_{2} / d_{1}\right)=1$.

We may extract the following immediate corollary which is more explicit when the Sylow 2-subgroup $C_{F}(2)$ of the ideal class group $C_{F}$ is cyclic.

Corollary. Let $F$ be as in the theorem with $D \equiv 3(\bmod 8)$. Suppose $C_{F}(2)$ is cyclic. Then, every integer in $F$ is a sum of three integer squares if and only if $D$ satisfies any of the three conditions: (a) $D$ is a prime; (b) $D=p q, p, q$ primes, $(p / q)=-1 ;$ and $(\mathrm{c}) D=p q, p, q$ primes, $(p / q)=1$, and $p \equiv 1(\bmod 8), q \equiv 3(\bmod 8)$.

We now see that all the previously known results are recaptured. Results (i) and (ii) are covered by the corollary. As for (iv), since the only prime factors of $D$ are those congruent to either 1 or $3(\bmod 8)$, no such proper factorizations as required by the theorem are possible. To see result (iii), suppose $q_{1} \equiv q_{2} \equiv 7(\bmod 8)$. Then $d_{1}$ can be either $q_{1}, q_{2}, q_{1} q_{3}$, or $q_{2} q_{3}$ so that violating condition (2) of the Theorem forces result (iii).

Next, we observe that since -1 is a sum of 2 squares in $F$ the quadratic space associated to the form $I_{3}=X^{2}+Y^{2}+Z^{2}$ must be isotropic, and therefore, the theoretical machinery derived in $[\mathbf{1}]$ may be applied. In particular, we can extract the following special cases of the theorems in $[1, \S \S 1,2]$ for the genus of $I_{3}$ over $F=\mathbf{Q}(\sqrt{-D})$.

FACT I. A nonzero integer $c$ of $F$ is an exceptional integer for the genus of $I_{3}$ (i.e., $c$ is representable by some, but not by every form in the genus) if and only if.

(I.1) $-c$ is a nonsquare in $F$;

(I.2) $-c$ is a square in $F_{2}$;

(I.3) $F(\sqrt{-c}) / F$ is unramified;

(I.4) writing $(c)=\left(\mathfrak{p}_{1}^{a_{1}} \cdots \mathfrak{p}_{t}^{a_{t}}\right)^{2}, a_{j}>0$, then at each $j$ the Artin symbol

$$
\left(\frac{F(\sqrt{-c}) / F}{\mathfrak{p}_{j}}\right)=1 \text {. }
$$

FACT $\Pi$. A nonzero integer $c$ of $F$ is an exceptional integer for $I_{3}$ (i.e., some form in the genus of $I_{3}$, but not $I_{3}$ itself, represents $c$ ) if and only if.

(II.1) $-c$ is a nonsquare in $F$;

(II.2) every prime dividing $(2 c)$ splits in $F(\sqrt{-c})$;

(II.3) $F(\sqrt{-c}) / F$ is unramified;

(II.4) if $\chi\left(I_{3}\right)=\tilde{A} C_{F}^{2}$ then $\tilde{A}$ is not a norm from $F(\sqrt{-c})$. 
Here $\tilde{A}$ denotes the ideal class of $A$. For the definition of the $\chi$-invariant, see [4, p. 329]. For our purpose here, it suffices to know that $\chi\left(I_{3}\right)=\tilde{A} C_{F}^{2}$ where $A$ is the coefficient ideal of any isotropic vector.

REMARK. Fact II solves the finer problem of whether a particular algebraic integer $c$ in $F$ is or is not a sum of 3 integer squares. This can be exploited to pinpoint explicitly an integer which is not a sum of 3 integer squares. For example, take the cyclic Sylow 2-subgroup case where $D=p q, p, q$ primes, $(p / q)=1, p \equiv$ $5(\bmod 8)$ and $q \equiv 7(\bmod 8)$. One checks that $c=q$ is an exception for $I_{3}$.

PRoOF of TheOREM. View the form $I_{3}$ as a free ternary lattice with orthonormal basis $\left\{e_{1}, e_{2}, e_{3}\right\}$. If $v$ is an isotropic vector with $v=a_{1} e_{1}+a_{2} e_{2}+a_{3} e_{3}$, then the coefficient ideal $A$ of $v$ in $I_{3}$ is given by

$$
\left(a_{1}^{-1}\right) \cap\left(a_{2}^{-1}\right) \cap\left(a_{3}^{-1}\right)=\left(a_{1}\right)+\left(a_{2}\right)+\left(a_{3}\right) .
$$

See [5, p. 212]. Let $R$ denote the ring of algebraic integers in $F$. Then, $\sum_{i=1}^{3} a_{i} R$ is generated by any two of the $a_{i}$. To see this, we localize and assume $R$ is a discrete valuation ring. Put $a R=\sum_{i=1}^{3} a_{i} R$ and $a_{i}=a t_{i}$. We may assume that not all $a_{i}$ are zero so that one of $t_{i}$ is a unit. But, $\sum t_{i}^{2}=0$. So, at least two of the $t_{i}$ must be units which proves the claim.

Suppose $r, s, t, w \in \mathbf{Z}$ satisfy the equation $D r^{2}=s^{2}+t^{2}+w^{2}$. Then,

$$
\left(s^{2}+t^{2}\right)^{2}+(s w+t r \sqrt{-D})^{2}+(t w-s r \sqrt{-D})^{2}=0
$$

gives rise to an isotropic vector. Hence, $\chi\left(I_{3}\right)=\tilde{A} C_{F}^{2}$ where $A=\left(s^{2}+t^{2}\right) R+$ $(s w+\operatorname{tr} \sqrt{-D}) R$.

Consider the binary quadratic form $f(X, Y)=-2 X^{2}+2 X Y+((D-1) / 2) Y^{2}$. Since $D \equiv 3(\bmod 8)$, this form is primitive. Hence, it represents an odd prime $p$ prime to any fixed integer of our choice (see e.g. Dickson's History, Vol. I, p. 417). We take $p$ prime to $D$. As $2 p=2 f(x, y)=-(2 x-y)^{2}+D y^{2}=-1+3(\bmod 8), p \equiv 1$ $(\bmod 4)$. Set $2 p=s^{2}+t^{2}, 2 x-y=w$ and $y=r$. Then, $D r^{2}=s^{2}+t^{2}+w^{2}=2 p+w^{2}$ and $\chi\left(I_{3}\right)=\tilde{A} C_{F}^{2}$, where

$$
A=p R+\left(\frac{s w+t r \sqrt{-D}}{2}\right) R
$$

Now,

$$
\begin{aligned}
N_{F / \mathbf{Q}}(A) & =\left(p R+\left(\frac{s w+t r \sqrt{-D}}{2}\right) R\right)\left(p R+\left(\frac{s w-t r \sqrt{-D}}{2}\right) R\right) \\
& =p\left(p R+s w R+\left(\frac{w^{2}+t^{2}}{2}\right) R+\left(\frac{s w+t r \sqrt{-D}}{2}\right) R\right)=p R
\end{aligned}
$$

since $p R+s w R+\left(\left(w^{2}+t^{2}\right) / 2\right) R=R$. We conclude, therefore, that $A$ is a prime ideal of norm $p \mathbf{Z}$.

Suppose $c$ is an exceptional integer for $I_{3}$, then it is surely exceptional for the genus of $I_{3}$. Set $(c)=B^{2}$ as in (I.4). Then $\tilde{B}$ contains an ambiguous ideal $E$. Write $B=b E$ and $E^{2}=\left(d_{1}\right)$, where $d_{1} \mid D$ and $c=u d_{1} b^{2}$ for some unit in $R$. We may assume clearly that $D>3$. Hence, $u= \pm 1$. Replacing $d_{1}$ by $-d_{1}$, if needed, we may suppose that $c=d_{1} b^{2}$. By (I.1), $d_{1} \neq-1, D$ and $d_{1} \equiv 3(\bmod 4)$ by (I.2). Since 
$F(\sqrt{-c})=F\left(\sqrt{-d_{1}}\right)=F\left(\sqrt{d_{2}}\right), d_{1} d_{2}=D$, condition (I.4) implies that

$$
\left(\frac{F\left(\sqrt{d_{2}}\right) / F}{E}\right)=\left(\frac{d_{2}}{\left|d_{1}\right|}\right)=1 \text {. }
$$

Next, condition (II.4)

$$
\left(\frac{F \sqrt{-c}) / F}{A}\right)=\left(\frac{-d_{1}}{p}\right)=\left(\frac{-2}{\left|d_{1}\right|}\right)=-1 .
$$

Conversely, assume there is a factorization $D=d_{1} d_{2}$ satisfying $d_{1} \equiv 3(\bmod 4)$, $d_{1} \neq-1, D,\left(d_{2} /\left|d_{1}\right|\right)=1$ and $\left(-2 /\left|d_{1}\right|\right)=-1$. Set $\left(d_{1}\right)=E^{2}$ and choose a prime ideal $P$ in $\tilde{E}$ and write $P=b E$ and $c=d_{1} b^{2}$. Then $(c)=P^{2}$. Clearly, $-c$ is a nonsquare in $F$. Since $d_{1} \equiv 3(\bmod 4),-c$ is a square in $F_{2}$. Condition (П.3) is a consequence of the equalities $F(\sqrt{-c})=F\left(\sqrt{-d_{1}}\right)=F\left(\sqrt{d_{2}}\right)$. Hence,

$$
\left(\frac{F(\sqrt{-c}) / F}{P}\right)=\left(\frac{F\left(\sqrt{d_{2}}\right) / F}{E}\right)=\left(\frac{d_{2}}{\left|d_{1}\right|}\right)=1 .
$$

So, we see (П.2) is satisfied. Finally, we note that $\left(-2 /\left|d_{1}\right|\right)=-1$ implies condition (II.4) is also met. This proves $c$ is an exceptional integer for $I_{3}$.

Recapturing, we have proved that $I_{3}$ has an exceptional integer if and only if $D$ admits a factorization $D=d_{1} d_{2}$ where (i) $d_{1} \equiv 3(\bmod 4)$, (ii) $d_{1} \neq-1, D$, (iii) $\left(d_{2} /\left|d_{1}\right|\right)=1$ and (iv) $\left(-2 /\left|d_{1}\right|\right)=-1$. If we require the factorization of $D$ to be positive proper then we get exactly the conditions as stated in our Theorem.

\section{REFERENCES}

1. D. R. Estes and J. S. Hsia, Exceptional integers of some ternary quadratic forms, Adv. in Math. 45 (1982), 310-318.

2. H. Hasse, Darstellbarkeit von Zahlen durch quadratische Formen in einem beliebigen algebraischen Zahlkorper, J. Reine Angew. Math. 153 (1924), 113-130.

3. J. S. Hsia, Representations by integral quadratic forms over algebraic number fields, Queen's Papers in Pure and Appl. Math. 46 (1977), 528-537.

4.

5. O. T. O'Meara, Introduction to quadratic forms, Grundlehren der Math. Wiss., Springer-Verlag, New York, 1963.

6. P. Revoy, Sur les sommes de carrés dans un anneau, Ann. Sci. Univ. Besançon Math. (3) 11 (1979), 3-8.

Department of Mathematics, University of Southern California, Los Angeles, California 90089-1113

Department of Mathematics, Ohio State University, Columbus, Ohio 43210 